\title{
DETERMINATION OF THE LEADING COEFFICIENT $a(x)$ IN THE HEAT EQUATION $u_{t}=a(x) \Delta u$
}

\author{
BY \\ BEI HU (University of Notre Dame, Notre Dame, Indiana) \\ AND \\ HONG-MING YIN (University of Toronto, Toronto, Ontario, Canada)
}

\begin{abstract}
This note deals with the parabolic inverse problem of determination of the leading coefficient in the heat equation with an extra condition at the terminal. After introducing a new variable, we reformulate the problem as a nonclassical parabolic equation along with the initial and boundary conditions. The existence of a solution is established by means of the Schauder fixed-point theorem.
\end{abstract}

1. Introduction. Recently, considerable effort was made in dealing with inverse problems in partial differential equations. These inverse problems not only have the intrinsic mathematical interests, but also have a variety of applications in industry and engineering sciences. It is known that an inverse problem is not well-posed in general. An important task is to formulate the problem properly and to find the conditions that ensure its well-posedness. In the present work, we study the inverse problem of finding $a(x)>0$ and $u(x, t)$, which satisfy:

$$
\begin{aligned}
u_{t} & =a(x) \Delta u, \quad(x, t) \in Q_{T} ; \\
u(x, t) & =g(x, t), \quad(x, t) \in S_{T}=\partial \Omega \times[0, T] ; \\
u(x, 0) & =u_{0}(x), \quad x \in \Omega,
\end{aligned}
$$

along with an extra condition

$$
u(x, T)=u_{1}(x), \quad x \in \Omega,
$$

where $T>0$ is fixed and $Q_{T}=\Omega \times(0, T]$, where $\Omega$ is a bounded domain in $\mathbf{R}^{n}$.

When an unknown coefficient appears in the lower-order terms, various results are obtained in [1, 2, 4] (also see [7] and the references therein). The uniqueness of solution of the problem (1.1)-(1.4) was studied in [8]. In the present work we shall follow the idea of [4] to establish the existence for the problem (1.1)-(1.4). After

Received October 28, 1991.

1991 Mathematics Subject Classification. Primary 35R25, 35R30.

The first author is partially supported by National Science Foundation DMS-90-24986, USA. The second author is partially supported by NSERC Canada. 
introducing a new variable, we have a nonlinear parabolic equation with the involvement of a trace-type functional as the leading coefficient. To avoid the degeneracy of the equation, we construct appropriate auxiliary functions and deduce some a priori estimates. The Schauder fixed point is used to prove the existence.

2. The assumptions and the main result. Let $v(x, t)=u_{t}(x, t)$. Then the extra condition (1.4) implies that

$$
a(x)=\frac{v(x, T)}{\Delta u_{1}(x)},
$$

provided $\Delta u_{1}(x) \neq 0$.

Now we differentiate Eq. (1.1) with respect to $t$; then $v(x, t)$ satisfies:

$$
\begin{aligned}
v_{t} & =\frac{v(x, T)}{\Delta u_{1}(x)} \Delta v, \quad(x, t) \in Q_{T} ; \\
v(x, t) & =g_{t}(x, t), \quad(x, t) \in S_{T} ; \\
v(x, 0) & =k(x) v(x, T), \quad x \in \Omega,
\end{aligned}
$$

where

$$
k(x)=\frac{\Delta u_{0}(x)}{\Delta u_{1}(x)} .
$$

Because of the nonlocal term $v(x, T)$, Eq. (2.1) is nonclassical. Moreover, the initial condition (2.3) is not known. In the sequel a solution of problem (1.1)-(1.4) or (2.1)-(2.3) is always understood in the classical sense.

Property. The problems (1.1)-(1.4) and (2.1)-(2.3) are equivalent if $\Delta u_{1} \neq 0$ in $\bar{\Omega}$.

Indeed, we have seen that if $u(x, t), a(x)$ is a solution of problem (1.1)-(1.4), then $v(x, t)$ is a solution of problem (2.1)-(2.3). Conversely, assuming that $v(x, t)$ is a solution of problem (2.1)-(2.3), we easily verify that

$$
u(x, t)=u_{0}(x)+\int_{0}^{t} v(x, \tau) d \tau, \quad a(x)=\frac{v(x, T)}{\Delta u_{1}}
$$

is a solution of the inverse problem (1.1)-(1.4). Therefore, we shall investigate the problem (2.1)-(2.3).

Throughout this paper the following conditions are assumed:

$\mathrm{H}(1)$ The functions $u_{0}(x), u_{1}(x) \in C^{4+\alpha}(\bar{\Omega})$,

$$
\Delta u_{0}(x) \geq 0, \quad 0<\Delta u_{1}(x) \leq M_{0} \quad \text { in } \bar{\Omega} .
$$

$\mathbf{H}(2)$ The function $g(x, t) \in C^{4+\alpha, 2+\alpha / 2}\left(S_{T}\right)$, and

$$
\begin{gathered}
0<g_{0} \leq g_{t}(x, t) \leq G_{0}, \quad g_{t}(x, 0)=k(x) g_{t}(x, T) \quad \text { for } x \in \partial \Omega \\
\text { and } \frac{d^{2}}{T} \leq \frac{2 g_{0}}{M_{0} e^{3 / 2}},
\end{gathered}
$$

where $d=\operatorname{MD}(\Omega)$ is the minimum diameter of $\Omega$, i.e., the infimum of distances between pairs $\Pi_{1}, \Pi_{2}$ of parallel planes such that $\Omega$ is contained in the strip determined by $\Pi_{1}$ and $\Pi_{2}$. 
$\mathbf{H}(3)$ The function $k(x)$ satisfies

$$
0 \leq k(x) \leq \exp \left(\frac{g_{0} T}{e^{3 / 2} M_{0}} e^{-2 d}\right) .
$$

The essential difficulty lies in that Eq. (2.1) may be degenerate, i.e., $v(x, T)$ may become zero at some points in $\Omega$. This would easily be avoided by using the maximum principle if the initial and the boundary data were uniformly positive; however, our initial condition is given by a relation between the initial and final states. The condition $\mathrm{H}(2)$ is physically reasonable since we require that $a(x)$ is positive, which is equivalent to saying that $v(x, T)>0$ on $\Omega$. This is the case if the surrounding temperature is high enough.

The main result is

TheOREM. Under the conditions $\mathrm{H}(1)-\mathrm{H}(3)$, the problem (1.1)-(1.4) admits a solution.

3. Proof. We shall use the Schauder fixed-point theorem to prove the result.

Proof of Theorem. Without loss of generality, we may assume that $0 \in \partial \Omega$ and that $\Omega$ lies in the strip $0 \leq x_{1} \leq d$. Let

$$
\begin{aligned}
K=\left\{w(x) \in C^{\alpha}(\bar{\Omega}): k_{0} \leq\right. & w(x) \leq G_{0}\left(e^{2 d}-e^{x_{1}}\right) \text { for } x \in \Omega, \\
w(x) & \left.=\frac{g_{t}(x, 0)}{k(x)} \text { for } x \in \partial \Omega \text { and }\|w\|_{C^{\alpha}(\bar{\Omega})} \leq k_{1}\right\},
\end{aligned}
$$

where the positive constants $k_{0}$ and $k_{1}$ will be specified later.

Obviously, each $w(x)$ in $K$ is bounded from above. For each $w(x) \in K$, we consider the problem:

$$
\begin{aligned}
v_{t} & =\frac{w(x)}{\Delta u_{1}(x)} \Delta v, & & (x, t) \in Q_{T} ; \\
v(x, t) & =g_{t}(x, t), & & (x, t) \in S_{T}, \\
v(x, 0) & =k(x) w(x), & & x \in \Omega .
\end{aligned}
$$

The standard theory of parabolic equations (cf. [6]) implies the problem admits a unique classical solution

$$
v(x, t ; w) \in C\left(\bar{Q}_{T}\right) \cap C^{2+\alpha, 1+\alpha / 2}\left(Q_{T}\right) .
$$

Moreover, that for any $t_{0}>0\left(t_{0}<T\right)$,

$$
v(x, t ; w) \in C^{2+\alpha, 1+\alpha / 2}\left(\bar{Q}_{T t_{0}}\right),
$$

where $Q_{T t_{0}}=Q_{T} \cap\left\{(x, t): x \in \bar{\Omega}, t_{0} \leq t \leq T\right\}$ and

$$
\|v\|_{C^{2+\alpha, 1+\alpha / 2}\left(\bar{Q}_{T t_{0}}\right)} \leq C_{0},
$$

where $C_{0}$ depends only on $k_{0}, k_{1}$ and known data. Also, by Krylov-Safanov's $C^{\alpha}$ estimate, $\|v\|_{C^{\alpha, \alpha / 2}\left(\bar{Q}_{T_{t_{0}}}\right)} \leq C_{1}$, where $C_{1}$ depends only on $k_{0}$ and known data and is independent of $k_{1}$. Hence we can take $k_{1}=C_{1}$. 
Now we define a mapping $M$ from $K$ into $C^{2+\alpha}(\bar{\Omega})$ as follows:

$$
M: w \in K \rightarrow v(x, T ; w) \in C^{2+\alpha}(\bar{\Omega}) \subset C^{\alpha}(\bar{\Omega}),
$$

where $v(x, t ; w)$ is the solution of problem (3.1)-(3.3).

We first show that $M$ is a continuous mapping from $C^{\alpha}(\bar{\Omega})$ to $C^{\alpha}(\bar{\Omega})$. Let $\left\{w_{n}(x)\right\} \subset K$ with $w_{n}(x) \rightarrow w(x)$ in $C^{\alpha}(\bar{\Omega})$ as $n$ tends to infinity. Let $v_{n}(x, t)$ and $v(x, t)$ be the corresponding solutions of Eqs. (3.1)-(3.3), respectively. Then the function $U(x, t)=v(x, t)-v_{n}(x, t)$ satisfies

$$
\begin{gathered}
L U=U_{t}-\frac{w(x)}{\Delta u_{1}(x)} \Delta U=\frac{\Delta v_{n}}{\Delta u_{1}}\left(w-w_{n}\right), \quad(x, t) \in Q_{T} ; \\
U(x, t)=0 \quad \text { on } S_{T} ; \quad U(x, 0)=k(x)\left[w(x)-w_{n}(x)\right] \quad \text { on } \Omega .
\end{gathered}
$$

By Green's representation, we have

$$
U(x, t)=\int_{\Omega} G(x, y ; t, 0) k(y)\left[w(y)-w_{n}(y)\right] d y+\int_{0}^{t} \int_{\Omega} G \frac{\Delta v_{n}}{\Delta u_{1}}\left[w-w_{n}\right] d y d \tau,
$$

where $G(x, y ; t, \tau)$ is the Green's function corresponding to the operator $L$. It follows by Eq. (3.4) that

$$
\max _{\bar{Q}_{T}}|U(x, t)| \leq C_{1}\left\|w-w_{n}\right\|_{0}+C_{1}\left\|w-w_{n}\right\|_{0} \rightarrow 0
$$

as $n \rightarrow \infty$. Using the Schauder estimate on $Q_{T t_{0}}$, we have

$$
\|U\|_{C^{2+\alpha, 1+\alpha / 2}\left(\bar{Q}_{T_{t_{0}}}\right)} \leq C\left[\|U\|_{0}+\left\|w-w_{n}\right\|_{C^{\alpha}(\bar{\Omega})}\right] \rightarrow 0
$$

as $n \rightarrow \infty$. In particular, as $n \rightarrow \infty$,

$$
\left\|v(x, T)-v_{n}(x, T)\right\|_{C^{\alpha}(\Omega)} \rightarrow 0 .
$$

The compactness of $M$ is clear since the embedding operator from $C^{2+\alpha}(\bar{\Omega})$ into $C^{\alpha}(\bar{\Omega})$ is compact. In order to apply the Schauder fixed-point theorem, it remains to prove that the mapping $M$ maps $K$ into itself.

Note that for $x \in \partial \Omega, v(x, 0)=k(x) w(x)=g_{t}(x, 0)$, it follows by $\mathrm{H}(2)$ that for $x \in \partial \Omega$

$$
v(x, T)=g_{t}(x, T)=\frac{g_{t}(x, 0)}{k(x)} .
$$

We shall next construct a subsolution for $v(x, t) \equiv v(x, t ; w)$. Let $\lambda=M_{0} / k_{0}$. For $x=\left(x_{1}, x_{2}, \ldots, x_{n}\right) \in \bar{\Omega}$, we introduce an auxiliary function

$$
\psi(x, t)=\frac{C^{*}}{\sqrt{t}} \exp \left(-\frac{\lambda\left(x_{1}-\xi_{1}\right)^{2}}{4 t}\right),
$$

where $\xi=\left(\xi_{1}, 0, \ldots, 0\right)$ is a fixed point, which lies in the outside of $R^{n} \backslash \bar{\Omega}$ and $C^{*}$ is a positive constant to be determined later. Then

$$
\psi_{t}(x, t)=C^{*}\left[-\frac{1}{2}+\frac{\lambda\left(x_{1}-\xi_{1}\right)^{2}}{4 t}\right] \frac{1}{\sqrt{t^{3}}} \exp \left(-\frac{\lambda\left(x_{1}-\xi_{1}\right)^{2}}{4 t}\right) .
$$


We choose $\xi$ such that for all $x=\left(x_{1}, \ldots, x_{n}\right) \in \Omega$,

$$
D \leq\left|x_{1}-\xi_{1}\right| \leq 2 D
$$

this is possible if we choose $D$ such that $D \geq d$. With the above choice of $\xi$, we have $\psi_{t}(x, t) \geq 0$ on $Q_{T}$ if we choose $D$ such that $D^{2} \lambda=2 T$. By a direct calculation, we see

$$
\Delta \psi=\lambda C^{*}\left[-\frac{1}{2}+\frac{\lambda\left(x_{1}-\xi_{1}\right)^{2}}{4 t}\right] \frac{1}{\sqrt{t^{3}}} \exp \left(-\frac{\lambda\left(x_{1}-\xi_{1}\right)^{2}}{4 t}\right) .
$$

It follows that

$$
\Delta \psi=\frac{1}{\lambda} \psi_{t} \geq 0
$$

Hence

$$
\begin{aligned}
\psi_{t}-\frac{w(x)}{\Delta u_{1}(x)} \Delta \psi & \leq \psi_{t}-\frac{k_{0}}{M_{0}} \Delta \psi \\
& =\psi_{t}-\frac{1}{\lambda} \Delta \psi=0
\end{aligned}
$$

Moreover, since $\xi \notin \bar{\Omega}$, we have, for all $x \in \Omega$,

$$
\psi(x, 0)=\lim _{t \rightarrow 0} \psi(x, t)=0 .
$$

Furthermore, on $S_{T}$, as $\left|x_{1}-\xi_{1}\right| \geq D$, we have

$$
\begin{aligned}
\psi(x, t) & \leq \frac{C^{*}}{\sqrt{t}} \exp \left(-\frac{\lambda D^{2}}{4 t}\right) \\
& \leq \frac{C^{*}}{\sqrt{T}} \sqrt{\frac{T}{t}} \exp \left(-\frac{\lambda D^{2}}{4 T} \frac{T}{t}\right) \\
& \leq \frac{C^{*}}{\sqrt{T}} \sup _{0<s<\infty}\left[\sqrt{s} \exp \left(-\frac{s}{2}\right)\right] \\
& =\frac{C^{*}}{\sqrt{T}} \frac{1}{\sqrt{e}} \\
& \leq g_{0},
\end{aligned}
$$

if we choose $C^{*}=\sqrt{e} \sqrt{T} g_{0}$. It follows that

$$
\psi(x, t) \leq g_{t}(x, t) \text { on } S_{T} .
$$

By the comparison principle, one obtains

$$
v(x, t) \geq \psi(x, t), \quad(x, t) \in \bar{Q}_{T} .
$$

In particular, on $\bar{\Omega}$,

$$
\begin{aligned}
v(x, T) & \geq \psi(x, T) \\
& \geq \sqrt{e} g_{0} \exp \left(-\frac{\lambda D^{2}}{T}\right) \\
& \geq \frac{1}{\sqrt{e^{3}}} g_{0} .
\end{aligned}
$$


Therefore, if we take $k_{0}=e^{-3 / 2} g_{0}$, then we have, on $\bar{\Omega}$,

$$
M w=v(x, T ; w) \geq k_{0} \text { for all } x \in \bar{\Omega} .
$$

With our choice of the constants $D, \lambda$, and $k_{0}$ and also using assumption $\mathrm{H}(2)$, we get

$$
D=\sqrt{\frac{2 T}{\lambda}}=\sqrt{\frac{2 T k_{0}}{M_{0}}}=\sqrt{\frac{2 T g_{0}}{M_{0} e^{3 / 2}}} \geq d,
$$

which is exactly what we assumed in the proof.

To show $v(x, T) \leq G_{0}\left(e^{2 d}-e^{x_{1}}\right)$, we introduce another auxiliary function:

$$
\varphi(x, t)=G_{0} e^{\gamma(T-t)}\left[e^{2 d}-e^{x_{1}}\right] .
$$

Then

$$
\begin{aligned}
\varphi_{t}-\frac{w(x)}{\Delta u_{1}(x)} \Delta \varphi & =G_{0} e^{\gamma(T-t)}\left[-\gamma\left(e^{2 d}-e^{x_{1}}\right)+\frac{w(x)}{\Delta u_{1}(x)} e^{x_{1}}\right] \\
& \geq G_{0} e^{\gamma(T-t)}\left(-\gamma e^{2 d}+\frac{k_{0}}{M_{0}} e^{x_{1}}\right) \\
& \geq G_{0} e^{\gamma(T-t)}\left(-\gamma e^{2 d}+\frac{k_{0}}{M_{0}}\right) \\
& =0
\end{aligned}
$$

if we choose $\gamma=\left(k_{0} / M_{0}\right) e^{-2 d}$. Recalling the definition for $k_{0}$, we conclude that $e^{\gamma T}=\exp \left(\left(g_{0} T / e^{3 / 2} M_{0}\right) e^{-2 d}\right)$. Thus, for $x \in \Omega$,

$$
\begin{aligned}
\varphi(x, 0) & =e^{\gamma T} G_{0}\left(e^{2 d}-e^{x_{1}}\right) \\
& \geq e^{\gamma T} w(x) \\
& \geq k(x) w(x) \quad[\text { by assumption } \mathrm{H}(3)] \\
& =v(x, 0) .
\end{aligned}
$$

On the boundary $S_{T}$,

$$
\begin{aligned}
\varphi(x, t) & \geq G_{0}\left(e^{2 D}-e^{x_{1}}\right) \\
& \geq G_{0}=\max \left|g_{t}\right| \\
& \geq v(x, t) .
\end{aligned}
$$

Again by the comparison principle, we have

$$
v(x, t) \leq \varphi(x, t) \text { on } \bar{Q}_{T} .
$$

It follows that

$$
v(x, T) \leq G_{0}\left(e^{2 D}-e^{x_{1}}\right) .
$$

Thus, the mapping $M$ is from $K$ into itself. By the Schauder fixed-point theorem, the mapping $M$ admits a fixed point, which is a solution of the problem (2.1)-(2.3). This completes our proof. 


\section{REFERENCES}

[1] N. Ya. Beznoshchenko, Sufficient conditions for the existence of solutions of the problem in the determination of coefficients of the leading derivatives of parabolic equations, Differentsial'nye Uravneniya 19, 1908-1915 (1983)

[2] J. R. Cannon and R. E. Ewing, Determination of a source term in a linear parabolic partial differential equation, J. Appl. Math. Phys. 27, 275-286 (1976)

[3] J. R. Cannon and H. M. Yin, A class of nonclassical parabolic problems, J. Differential Equations 79, 266-288 (1989)

[4] J. M. Chadam and H. M. Yin, Determination of an unknown function in a parabolic equation with an overspecified condition, Math. Methods Appl. Sci. 13, 421-430 (1990)

[5] D. Colton, R. E. Ewing, and W. Rundell, Inverse problems in partial differential equations, SIAM Proc., Philadelphia, 1990

[6] A. Friedman, Partial differential equations of parabolic type, Prentice Hall, Englewood Cliffs, NJ, 1964

[7] V. Isakov, Inverse source problems, Math. Surveys Monographs, no. 34, Amer. Math. Soc., Providence, RI, 1990

[8] W. Rundell, The determination of a parabolic equation from the initial and final data, Proc. Amer. Math. Soc. 99, 637-642 (1987) 